\title{
A fundamental plane for field star-forming galaxies $\star$
}

\author{
M. A. Lara-López ${ }^{1,2}$, J. Cepa ${ }^{1,2}$, A. Bongiovanni ${ }^{1,2}$, A. M. Pérez García ${ }^{1,2}$, A. Ederoclite ${ }^{1,2}$, H. Castañeda ${ }^{1,3}$, \\ M. Fernández Lorenzo ${ }^{1,2}$, M. Pović ${ }^{1,2}$, and M. Sánchez-Portal ${ }^{4}$ \\ 1 Instituto de Astrofísica de Canarias, 38200 La Laguna, Spain \\ e-mail: mall@iac.es \\ 2 Departamento de Astrofísica, Universidad de la Laguna, Spain \\ 3 Departamento de Física, Escuela Superior de Física y Matemática, IPN, Mexico D.F., Mexico \\ ${ }^{4}$ Herschel Science Center, INSA/ESAC, Madrid, Spain
}

Received 15 April 2010 / Accepted 24 September 2010

\section{ABSTRACT}

\begin{abstract}
Context. Star formation rate (SFR), metallicity, and stellar mass are among the most important parameters of star-forming (SF) galaxies characterizing their formation and evolution. They are known to be related to each other both at low and high redshift in the mass-metallicity, mass-SFR, and metallicity-SFR relations.

Aims. We demonstrate the existence of a plane in a 3D parameter space defined by the axes SFR $\left[\log (S F R)\left(M_{\odot} \mathrm{yr}^{-1}\right)\right]$, gas metallicity $[12+\log (\mathrm{O} / \mathrm{H})]$, and stellar mass $\left[\log \left(M_{\mathrm{star}} / M_{\odot}\right)\right]$ of SF galaxies.

Methods. We used SF galaxies from the "main galaxy sample" of the Sloan Digital Sky Survey-Data Release 7 (SDSS-DR7) in the redshift range $0.04<z<0.1$ and $r$-magnitudes between 14.5 and 17.77. Metallicities, SFRs, and stellar masses were taken from the Max-Planck-Institute for Astrophysics-John Hopkins University (MPA-JHU) emission-line analysis database.

Results. From a final sample of 32575 galaxies, we find for the first time a fundamental plane for field galaxies relating the SFR, gas metallicity, and stellar mass for SF galaxies in the local universe. One of the applications of this plane would be to estimate stellar masses from SFR and metallicity. High redshift data from the literature at redshift $\sim 0.85,2.2$, and 3.5 , do not show evidence of evolution in this fundamental plane.
\end{abstract}

Key words. galaxies: fundamental parameters - galaxies: abundances - galaxies: starburst - galaxies: star formation

\section{Introduction}

Relations between important properties of astrophysical objects often lead to the discovery of the so-called fundamental planes when three parameters are involved. The fundamental plane (FP) for elliptical galaxies (Djorgovski \& Davis 1987; Dressler et al. 1987), relates their luminosity, velocity dispersion (dynamics), and scale length (morphology). This FP represents an important tool to investigate the properties of early-type and dwarf galaxies, to perform cosmological tests, and compute cosmological parameters. It is also an important diagnostic tool for galaxy evolution and mass-to-light $(M / L)$ variations with redshift.

Fundamental planes have also been defined for globular clusters (Meylan \& Heggie 1997) and galaxy clusters (Schaeffer et al. 1993; Adami et al. 1998). The parameter space of globular clusters, elliptical galaxies, and galaxy clusters is properly described by a geometrical plane $L \propto R^{\alpha} \sigma^{\beta}$, where $L$ is the optical luminosity of the system, $R$ is a measure of the size scale, $\sigma$ is the velocity dispersion of the system, and $\alpha$ and $\beta$ are free parameters. The FP for globular clusters, elliptical galaxies, and galaxy clusters have very similar slopes, which means that, accounting for differences in zero points, a single FP with a range of about nine orders of magnitude in luminosity can be defined (Schaeffer et al. 1993; Ibarra \& López-Cruz 2009).

The FP that we introduce here relates three fundamental parameters: the SFR $\left[\log (S F R)\left(M_{\odot} \mathrm{yr}^{-1}\right)\right]$, gas metallicity $[12+\log (\mathrm{O} / \mathrm{H})]$, and stellar mass $\left[\log \left(M_{\mathrm{star}} / M_{\odot}\right)\right]$ of field SF galaxies. All these variables have been related in the past by

* Appendix $\mathrm{A}$ is only available in electronic form at http://www . aanda.org the mass-metallicity $(M-Z)$ relation (Lequeux et al. 1979), the mass-SFR relation (Brinchmann et al. 2004), and the metallicitySFR relation (Lara-López et al. 2010; López-Sánchez 2010). Some authors have also studied the inter-dependence of those variables (e.g. Hoopes et al. 2007; Ellison et al. 2008; Mannucci et al. 2010). In the present work, we propose the generalization of those relations defining a plane formed by a linear combination of two of those variables with respect to the third one.

The $M-Z$ relation connects the mass to the metallicity of galaxies, massive galaxies being found to have higher metallicities than less massive ones, and has been well established for the local universe $(z \sim 0.1)$ by the work of Tremonti et al. (2004) using SDSS data. The $M-Z$ relation has also been studied at low redshifts $z \sim 0.35$ (Lara-López et al. 2009a,b), at intermediate redshifts $z \sim 0.7$ (e.g., Rodrigues et al. 2008), and at high redshift $z \sim 2.2$ and $z \sim 3.5$ (Erb et al. 2006; Maiolino et al. 2008, respectively).

The stellar mass of SF galaxies is also related to the SFR, in the sense that more massive galaxies have higher SFRs (Brinchmann et al. 2004; Salim et al. 2005). However, Brinchmann et al. (2004) emphasized that at $\log \left(M_{\text {star }} / M_{\odot}\right) \gtrsim$ 10 , the distribution of SFRs broadens significantly and the correlation between stellar mass and SFR breaks down. At higher redshifts, Noeske et al. (2007) showed the existence of a "main sequence" for this relation over the redshift range $0.2<z<1.1$.

The metallicity and SFR of SF galaxies are weakly correlated, as can be seen in Fig. 1. However, and despite the high scatter, SFR increases with metallicity (Lara-López et al. 2010; López-Sánchez 2010). 

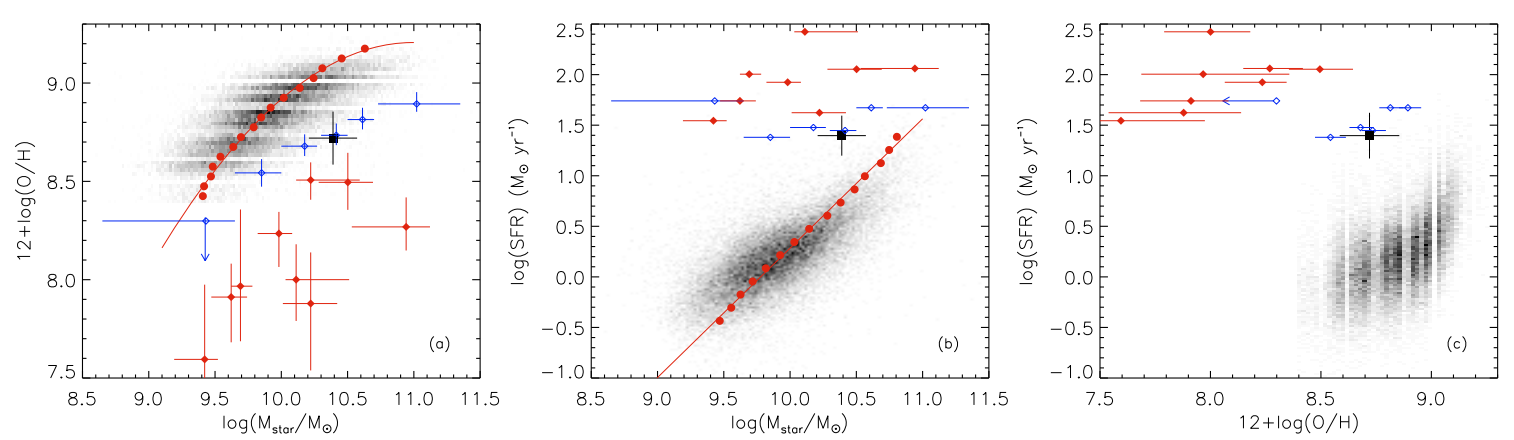

Fig. 1. In panels a), b), and c), the $M-Z$, stellar mass-SFR, and metallicity-SFR relations are shown respectively, the density plots are represented in bins of 0.02 dex. Red circles represent the median mass in bins of 0.05 dex in $12+\log (\mathrm{O} / \mathrm{H})$ and of 0.13 dex in $\log (S F R)$. Blue empty diamonds represent the Erb et al. (2006) data at $z \sim 2.2$, the bars of their data show the metallicity error and the stellar mass range of each bin, while the SFR errors are about $\sim 1.3$ dex. Red filled diamonds represent the sample of Maiolino et al. (2008) at $z \sim 3.5$, the bars of their data show the metallicity and mass errors. The black filled square represents the median of the data of Rodrigues et al. (2008) at $z \sim 0.85$ with its respective error bars. Solid lines in panels a) and b) represent a second and first order polynomial fits to the median values, respectively.

This paper is structured as follows. Section 2 describes the data selection as well as the measurement of the SFRs, metallicities and stellar masses provided by the MPA-JHU group ${ }^{1}$ and adopted in this work. In Sect. 3, we define the FP for field galaxies, and our conclusions are given in Sect. 4.

\section{Data processing and sample selection}

Our study was carried out using data for galaxies from SDSSDR7 (York et al. 2000; Abazajian et al. 2009). Data were taken with a $2.5 \mathrm{~m}$ telescope located at Apache Point Observatory (Gunn et al. 2006); additional technical details can be found in Stoughton et al. (2002).

We used the emission-line analysis of SDSS-DR7 galaxy spectra performed by the MPA-JHU group. From the full dataset, we only considered objects classified as galaxies in the "main galaxy sample" (Strauss et al. 2002) with apparent Petrosian $r$ magnitude in the range $14.5<r<17.77$ and redshift range $0.04<z<0.1$, which represent a complete sample in magnitude and redshift (e.g. Kewley \& Ellison 2008). The lower limit in redshift ensures that we cover $>20 \%$ of the galaxy light, which is the minimum required to avoid domination of the spectrum by aperture effects (Kewley et al. 2005). Following Kewley \& Ellison (2008) and Kobulnicky \& Zaritsky (1999), to obtain reliable metallicity estimates we selected galaxies with a signal-to-noise ratio (SNR) higher than 8 for the $\mathrm{H} \alpha, \mathrm{H} \beta$, [N II] $\lambda 6584,[\mathrm{O}$ II] $\lambda 3727,[\mathrm{O} \mathrm{III}] \lambda 5007$, and [S II] $\lambda \lambda 6717$, 6731 lines. However, using less restrictive criteria does not affect the relations derived here, but only increases their dispersion. For a detailed analysis of the line SNR, we refer to Brinchmann et al. (2004). Finally, SF galaxies were selected following the criterion given by Kauffmann et al. (2003a) for the BPT empirical diagnostic diagram $\log [\mathrm{O}$ III $] \lambda 5007 / \mathrm{H} \beta \leq 0.61 /\{\log ([\mathrm{N} \mathrm{II}] / \mathrm{H} \alpha)$ $0.05\}+1.3$. From this final sample of 32575 galaxies, metallicities, stellar masses, and SFRs used in the present work were obtained by the MPA-JHU group following the methods described below. Since field galaxies are the dominant population of this sample, the FP presented here would be representative of field galaxies, and differ from the known FP of elliptical and clusters of galaxies.

Metallicities were estimated statistically using Bayesian techniques according to Tremonti et al. (2004), based on simultaneous fits of all the most prominent emission lines ([O II], $\mathrm{H} \beta$, [O III], H $\alpha$, [N II], [S II]) using a model designed for the interpretation of integrated galaxy spectra (Charlot \& Longhetti 2001).

\footnotetext{
${ }^{1}$ http://www.mpa-garching.mpg.de/SDSS
}

Since the metallicities derived with this technique are discretly sampled, they exhibit small random offsets (see for details Tremonti et al. 2004). Any dependence of SFR on the estimated metallicity would be minor (Tremonti et al. 2004; Brinchmann et al. 2008). For this work, we selected galaxies with $12+\log (\mathrm{O} / \mathrm{H})>8.4$, corresponding to the upper branch of the $R_{23}$. However, galaxies with $12+\log (\mathrm{O} / \mathrm{H})<8.4$, corresponding to the lower branch of the $R_{23}$ calibration, are poorly sampled (e.g. Tremonti et al. 2004; Kewley \& Ellison 2008). Therefore, to avoid a systematic dispersion in the FP, and to work with a homogeneous sample, we selected galaxies with $12+\log (\mathrm{O} / \mathrm{H})>8.4$, which correspond to $\sim 99 \%$ of our SF sample.

Total stellar masses were estimated from fits to the photometry using the same modeling methodology as described in Kauffmann et al. (2003), with only small differences with respect to previous data released.

Finally, total SFRs for SF galaxies were inferred directly from the emission lines, based on the careful modeling discussed in Brinchmann et al. (2004), who modeled the emission lines in the galaxies following the Charlot \& Longhetti (2001) prescription, achieving a robust dust correction. The metallicity dependence of the case $\mathrm{B} \mathrm{H} \alpha / \mathrm{H} \beta$ ratio is also taken into account. The Brinchmann et al. (2004) method offers a more robust SFR estimate than using, for example, a fixed conversion factor between H $\alpha$ luminosity and SFR (e.g. Kennicutt 1998).

The FP presented in this study was initially identified by us using STARLIGHT data (Cid Fernandes et al. 2005; Mateus et al. 2006) for the sample described above, but estimating SFRs using the $\mathrm{H} \alpha$ luminosity and the Kennicutt (1998) relation, and metallicities following the calibration of Tremonti et al. (2004). Nevertheless, although the FP derived is the same, it is noteworthy that the plane has a lower scatter when using the robust SFR and metallicity estimations derived by the MPA-JHU group.

\section{The fundamental plane}

As mentioned in the introduction, the SFR, stellar mass, and gas metallicity of SF galaxies are related to each other. Their strong relation is evident when these data are plotted in a $3 \mathrm{D}$ space with orthogonal coordinate axes defined by these parameters. A careful inspection of this $3 \mathrm{D}$ representation shows the existence of a plane (see Appendix A).

The projection of galaxy data over any pair of the axes of this $3 \mathrm{D}$ space will reduce to the $M-Z$, metallicity-SFR, and stellar mass-SFR relations, as shown in Fig. 1. Tremonti et al. (2004) 


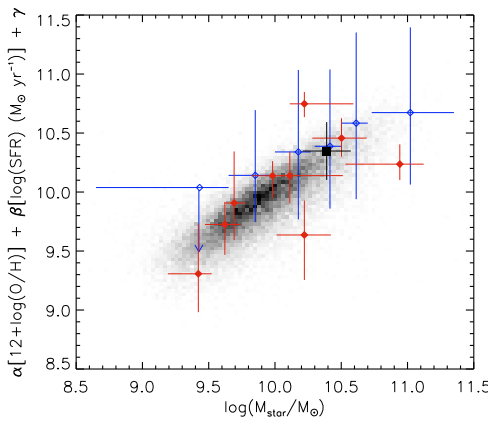

Fig. 2. FP for field SF galaxies. The stellar mass, in units of solar masses, is presented as a linear combination of $12+\log (\mathrm{O} / \mathrm{H})$ and $\log (S F R)$. Symbols follow the same code as in Fig. 1. Since the Maiolino et al. (2008) data do not have SFR errors, the vertical bars of their data show only the error in metallicity of the linear combination $[\alpha \Delta(12+\log (\mathrm{O} / \mathrm{H}))]$.

demonstrated that the metallicity of galaxies increases with the stellar mass in a relatively steep way from $10^{8.5}$ to $10^{10.5} M_{\odot}$, but flattens above $10^{10.5} M_{\odot}$ (see Fig. 1a). Kewley \& Ellison (2008) recalibrated the $M-Z$ relation of Tremonti et al. (2004) using the completeness criteria of redshift and magnitude given in Sect. 2. However, since the Kewley \& Ellison (2008) relation (see Fig. 1.1 of that paper) does not accurately describe the low mass population, we fit a second-order polynomial to the median mass in metallicity bins of the $M-Z$ relation, $12+\log (\mathrm{O} / \mathrm{H})=a_{0}+a_{1}\left[\log \left(M_{\text {star }} / M_{\odot}\right)\right]+a_{2}\left[\log \left(M_{\text {star }} / M_{\odot}\right)\right]^{2}$, where $a_{0}=-25.93923, a_{1}=6.39283, a_{2}=-0.29071$, and $\sigma=0.26$. Regarding the mass-SFR relation (see Fig. 1b), the SFR increases with stellar mass up to $\sim 10^{10} M_{\odot}$ (Brinchmann et al. 2004), while for higher mass values the scatter increases (see Fig. 1b). We fitted a line to the median mass in $\log (S F R)$ bins of the mass-SFR relation given by $\log (S F R)=a_{0}+a_{1}\left[\log \left(M_{\text {star }} / M_{\odot}\right)\right]$, where $a_{0}=-12.50704$, $a_{1}=1.27909$, and $\sigma=0.27$. The $\sigma$ given is that of the horizontal axes $\left[\log \left(M_{\mathrm{star}} / M_{\odot}\right)\right]$ in both cases. However, for $12+\log (\mathrm{O} / \mathrm{H}) \lesssim 8.9$, the SFR is not strongly correlated with metallicity (see Fig. 1c), whereas for higher metallicity values, the SFR increases rapidly. Therefore, since the metallicity-SFR relation does not correlate at all, this leads us towards a possible solution: a linear combination of the metallicity and SFR as a function of the stellar mass.

We generated least squares fits of first and second order polynomials combining two of the variables as a function of the third one, trying all the possible combinations. As expected, the optimal fit corresponds to a linear combination of the SFR and metallicity that yields the stellar mass (see Fig. 2). The FP for field SF galaxies is then defined by

$\log \left(M_{\mathrm{star}} / M_{\odot}\right)=\alpha[12+\log (\mathrm{O} / \mathrm{H})]+\beta\left[\log (S F R)\left(M_{\odot} \mathrm{yr}^{-1}\right)\right]+\gamma$

where $\alpha=1.122( \pm 0.008), \beta=0.474( \pm 0.004), \gamma=$ $-0.097( \pm 0.077)$, and $\sigma=0.16$. The sigma (standard deviation) given is that of the vertical axis of Fig. 2.

One of the uses of this FP would be to estimate the stellar masses from the metallicity and the SFR of emission-line galaxies. In analogy to the FP of elliptical galaxies, it is expected that this FP reduces the scatter of the stellar mass found using the $M-Z$ or mass-SFR relations. To compare the mass $\left(M_{\text {star }} / M_{\odot}\right)$ width of this FP with that of the $M-Z$ and mass-SFR relations, we estimated the 90th and $\sim 68$ th mass percentile around the fit to each relation, obtaining a mass width for the 90th percentile of $\sim 0.57, \sim 0.87$, and $\sim 0.92$ dex for the FP, $M-Z$, and mass-SFR relations, respectively. Using a $\sim 68$ th mass percentile, we found a mass range of $\sim 0.32, \sim 0.52$, and $\sim 0.53$ dex for the same relations. As observed, the scatter in the mass estimates inferred from the FP is $\sim 0.3$ and 0.2 dex smaller than that of the $M-Z$ relation for the 90 th and $\sim 68$ th mass percentiles, respectively, which means that the mass dispersion using the FP is reduced to $\sim 38 \%$ for a 68 th percentile.

The largest expected errors in the stellar mass estimates that would be obtained using this FP, without considering SFR and metallicity errors, would be about \pm 0.28 dex for a 90 th mass percentile, and \pm 0.16 dex for a $\sim 68$ th mass percentile ( $1 \sigma$ error). Therefore, since the FP is a well established linear relation, and its dispersion in mass is smaller than that of the $M-Z$ and massSFR relations, it is a useful tool for deriving stellar masses.

To identify possible signs of evolution in the FP, we used the data of Rodrigues et al. (2008) at $z \sim 0.85$, Erb et al. (2006) at $z \sim 2.2$, and Maiolino et al. (2008) at $z \sim 3.5$, as shown in Figs. 1 and 2. The Erb et al. (2006) metallicity estimates, derived using the N2 method and the calibration of Pettini \& Pagel (2004), and the Maiolino et al. (2008) estimates, derived using the calibrations of Kewley \& Dopita (2002), were recalibrated to the $R_{23}$ method using the conversions of Kewley \& Ellison (2008). The Erb et al. (2006) data use the Bruzual \& Charlot (2003) population synthesis models and a Chabrier (2003) IMF. According to Bruzual \& Charlot (2003), the Chabrier (2003) and Kroupa (2001) IMF, used by Erb et al. (2006) and in this study, respectively, yield practically identical $M / L$ ratios. However, since the stellar masses of both Maiolino et al. (2008) and Rodrigues et al. (2008) were estimated using a Salpeter (1955) IMF, we correct them, as indicated by Maiolino et al. (2008), by dividing their masses by a factor of 1.17 to make these data consistent with our assumed IMF.

In Fig. 1a, the well-known evolution in the $M-Z$ relation can be appreciated (e.g. Erb et al. 2006; Maiolino et al. 2008), while in Fig. 1b an important evolution in the $\log (S F R)$ of $\sim 1.5 \mathrm{dex}$ is observed for an intermediate mass of $10^{10.1} M_{\odot}$, as well as a flattening in the shape of the mass-SFR relation, already noted by Lara-López et al. (2010). The evolution in the $M-Z$ relation, as argued by Liang et al. (2006), is due to a decrease in the metal content of galaxies rather than an increase in their stellar mass. The evolution observed in the mass-SFR relation is also due to an increase in the SFR in galaxies. Therefore, the metallicitySFR relation shows evolution in both axes, as shown in Fig. 1c.

We apply Eq. (1) to these high redshift data. As observed in Fig. 2, the Erb et al. (2006) data suggest an evolution in the slope. However, the uncertainties in the measured parameters are too large. Moreover, if this change in slope were real, it would also be evident in the data of Maiolino et al. (2008), and it is not. Therefore, we conclude that current high redshift data do not provide clear evidence of evolution in the FP.

It is noteworthy that the main evolution in the $M-Z$ and mass-SFR relations is driven by the metallicity and SFR, respectively, rather than the stellar mass. Therefore, we would not expect a mass evolution in the mass projection of the FP, as can be appreciated in Fig. 2. This lack of evolution in our relation could be explained by the metallicity and SFR evolving in opposite directions, which would imply that high redshift galaxies have lower metallicity values but higher SFRs than the local sample. For example, those diferences could be, for an intermediate mass of $\sim 10^{10.1} M_{\odot}$, about $2.12 \mathrm{dex}$ in $\log (S F R)$, and $\sim 0.7 \mathrm{dex}$ in $12+\log (\mathrm{O} / \mathrm{H})$. This means that the high SFRs at higher redshifts would be compensated by their lower metallicities when the $\alpha$ and $\beta$ coefficients of Eq. (1). are taken into account.

Stellar mass is the physical fundamental parameter driving the SFR and metallicity of star forming galaxies. Both SFR and 
metallicity increase with mass, as shown in the $M-Z$ and massSFR relations of Fig. 1. A possible explanation is given by the well-known effect of downsizing (Cowie et al. 1996; Gavazzi \& Scodeggio 1996), in which the less massive galaxies form their stars later and on longer timescales than more massive systems. This implies that low mass galaxies have lower metallicities and higher specific SFRs. Since the star-formation-rate history drives the metal enrichment, downsizing correlates both parameters with mass. Their linear combination would then relate current star formation rate with its past history, increasing the accuracy of the mass determination by reducing the scatter in the relation.

The stellar mass of galaxies is usually estimated using the $z$-band magnitude, the spectral indices $D_{n}(4000)$ and $\mathrm{H} \delta_{\mathrm{A}}$, and by assuming an IMF, such as the method used by Kauffmann et al. (2003b). It is also possible to use sophisticated codes such as STARLIGHT (Cid Fernandes et al. 2005; Mateus et al. 2006), which fits an observed spectrum with a combination of simple stellar populations (SSPs) from the evolutionary synthesis models of Bruzual \& Charlot (2003), computed using a Chabrier (2003) IMF, and "Padova 1994" evolutionary tracks (Girardi et al. 1996). Moreover, the masses obtained must be corrected for aperture effects based on the differences between the total galaxy magnitude in the $r$ band, and the magnitude inside the fiber, assuming that the $M / L$ ratio does not depend on the radius (see Mateus et al. 2006, for details). Therefore, applying the FP presented here allows us to estimate stellar masses in an easier and reliable way than existing methods.

Finally, in an independent and parallel study, Mannucci et al. (2010) fit a 2D surface instead of a plane to the same variables, which also reduces the metallicity dispersion. However, one of the most important differences between that study and our present work is that we analyze a complete sample in redshift $(z<0.1)$ to avoid systematic effects (e.g. Lara-López et al. 2010). In addition, the plane fitted by ourselves also accounts for the data at higher redshifts, supporting the apparent evidence of no-evolution presented in this section, as explained above, which makes it invaluable for estimating the stellar masses of field galaxies even up to $z \sim 3.5$.

\section{Conclusions}

We have demonstrated the existence of a FP for field SF galaxies in the 3D space formed by the orthogonal coordinate axes $\log \left(M_{\text {star }} / M_{\odot}\right), \log (S F R)\left(M_{\odot} \mathrm{yr}^{-1}\right)$, and $12+\log (\mathrm{O} / \mathrm{H})$, three of the fundamental parameters of SF galaxies. All these variables have been related previously in pairs as with the $M-Z$, metallicity-SFR, and mass-SFR relations, but this is the first time that the correlation for all of them has been quantified.

The FP presented here allows us to estimate the stellar mass $\left[\log \left(M_{\mathrm{star}} / M_{\odot}\right)\right]$ of field galaxies as a linear combination of $12+\log (\mathrm{O} / \mathrm{H})$ and $\log (S F R)\left(M_{\odot} \mathrm{yr}^{-1}\right)$. The scatter in the mass estimates using the FP ( $1 \sigma$ error of 0.16$)$ is lower that that obtained using the $M-Z$ and mass-SFR relations.

The FP introduced here would be useful for deriving masses in spectroscopic surveys where the SFR and metallicity are estimated for emission-line galaxies, for example, using the $\mathrm{H} \alpha$ luminosity to estimate the SFR (e.g. Kennicutt 1998), and any of the metallicity methods in the literature, such as the $R_{23}$ (Pagel et al. 1979) or N2 (Denicoló et al. 2002) (see Kewley \& Ellison (2008) for a review). However, since this study has been carried out using emission-line galaxies, this FP will be useful only when both SFR and metallicity of galaxies can be estimated.

Within the errors, there is no evidence of an evolution in the local FP when applied to high redshift samples. This implies that this relation could be useful even at high redshifts, where measuring the continuum and absorption lines for fitting models would be more difficult and time consuming.

We therefore propose the use of this FP as an alternative tool to existing methods to determine the stellar mass of galaxies at low and high redshifts.

Acknowledgements. This work was supported by the Spanish Plan Nacional de Astronomía y Astrofísica under grant AYA2008-06311-C02-01. We especially thank C. Bertout, M. Walmsley, F. Moreno-Insertis, and our last referee for all their help. We thank J. Brinchmann for providing useful details about MPAJHU data. M. A. Lara-López is supported by a CONACyT and SEP Mexican fellowships.

\section{References}

Abazajian, K. N., Adelman-McCarthy, J. K., Agüeros, M. A., et al. 2009, ApJS, 182,543

Adami, C., Mazure, A., Biviano, A., et al. 1998, A\&A, 331, 493

Brinchmann, J., Charlot, S., White, S. D. M., et al. 2004, MNRAS, 351, 1151

Brinchmann, J., Pettini, M., \& Charlot, S. 2008, MNRAS, 385, 769

Bruzual, G., \& Charlot, S. 2003, MNRAS, 344, 1000

Chabrier, G. 2003, PASP, 115, 763

Charlot, S., \& Fall, S. M. 2000, ApJ, 539, 718

Charlot, S., \& Longhetti, M. 2001, MNRAS, 323, 887

Charlot, S., Kauffmann, G., Longhetti, M., et al. 2002, MNRAS, 330, 876 Cid Fernandes, R., Mateus, A., Sodré, L., et al. 2005, MNRAS, 358, 363 Cowie, L. L., Songaila, A., Hu, E. M., \& Cohen, J. G. 1996, AJ, 112, 839 Denicoló, G., Terlevich, R., \& Terlevich, E. 2002, MNRAS, 330, 69 Djorgovski, S., \& Davis, M. 1987, ApJ, 313, 59

Dressler, A., Lynden-Bell, D., Burstein, D., et al. 1987, ApJ, 313, 42 Ellison, S. L., Patton, D. R., Simard, L., et al. 2008, ApJ, 672, L107 Erb, D. K., Shapley, A. E., Pettini, M., et al. 2006, ApJ, 644, 813 Gavazzi, G., \& Scodeggio, M. 1996, A\&A, 312, L29

Girardi, L., Bressan, A., Chiosi, C., et al. 1996, A\&AS, 117, 113 Gunn, J. E., Siegmund, W. A., Mannery, E. J., et al. 2006, AJ, 131, 2332 Hoopes, C. G., Heckman, T. M., Salim, S., et al. 2007, ApJS, 173, 441 Ibarra, H. J., \& López-Cruz, O. 2009, Rev. Mex. Astron. Astrofis., 37, 106 Kauffmann, G., Heckman, T. M., Tremonti, C., et al. 2003a, MNRAS, 346, 1055 Kauffmann, G., Heckman, T. M., White, S. D. M., et al. 2003b, MNRAS, 341, 33

Kennicutt, R. C., Jr. 1998, ARA\&A, 36, 189

Kewley, L. J., \& Dopita, M. A. 2002, ApJS, 142, 35 Kewley, L. J., \& Ellison, S. L. 2008, ApJ, 681, 1183

Kewley, L. J., Jansen, R. A., \& Geller, M. J. 2005, PASP, 117, 227

Kobulnicky, H. A., \& Zaritsky, D. 1999, ApJ, 511, 118

Kroupa, P. 2001, MNRAS, 322, 231

Lara-López, M. A., Cepa, J., Bongiovanni, A., et al. 2009a, A\&A, 493, L5 Lara-López, M. A., Cepa, J., Bongiovanni, A., et al. 2009b, A\&A, 505, 529 Lara-López, M. A., Bongiovanni, A., Cepa, J., et al. 2010, A\&A, 519, A31 Lequeux, J., Peimbert, M., Rayo, J. F., et al. 1979, A\&A, 80, 155 Liang, Y. C., Hammer, F., \& Flores, H. 2006, A\&A, 447, 113 López-Sánchez, A. R. 2010, A\&A, in press [arXiv: 1005.0659] Maiolino, R., Nagao, T., Grazian, A., et al. 2008, A\&A, 488, 463 Mannucci, F., Cresci, G., Maiolino, R., et al. 2010, MNRAS [arXiv: 1005.0006 ]

Mateus, A., Sodré, L., Cid Fernandes, R., et al. 2006, MNRAS, 370, 721 Meylan, G., \& Heggie, D. C. 1997, A\&A Rev., 8, 1

Noeske, K. G., Weiner, B. J., Faber, S. M., et al. 2007, ApJ, 660, L43 Pagel, B. E. J., Edmunds, M. G., Blackwell, D. E., et al. 1979, MNRAS, 189, 95 Pettini, M., \& Pagel, B. E. J. 2004, MNRAS, 348, L59

Rodrigues, M., Hammer, F., Flores, H., et al. 2008, A\&A, 492, 371

Salim, S., Charlot, S., Rich, R. M., et al. 2005, ApJ, 619, L39

Salpeter, E. E. 1955, ApJ, 121, 161

Schaeffer, R., Maurogordato, S., Cappi, A., \& Bernardeau, F. 1993, MNRAS, 263, L21

Stoughton, C., Lupton, R. H., Bernardi, M., et al. 2002, AJ, 123, 485

Strauss, M. A., Weinberg, D. H., Lupton, R. H., et al. 2002, AJ, 124, 1810 Tremonti, C. A., Heckman, T. M., Kauffmann, G., et al. 2004, ApJ, 613, 898 York, D. G., Adelman, J., Anderson, J. E., Jr., et al. 2000, AJ, 120, 1579

Page 5 is available in the electronic edition of the journal at http://www . aanda.org 
M. A. Lara-López et al.: A fundamental plane for field star-forming galaxies

\section{Appendix A: Fundamental plane in a 3D space}

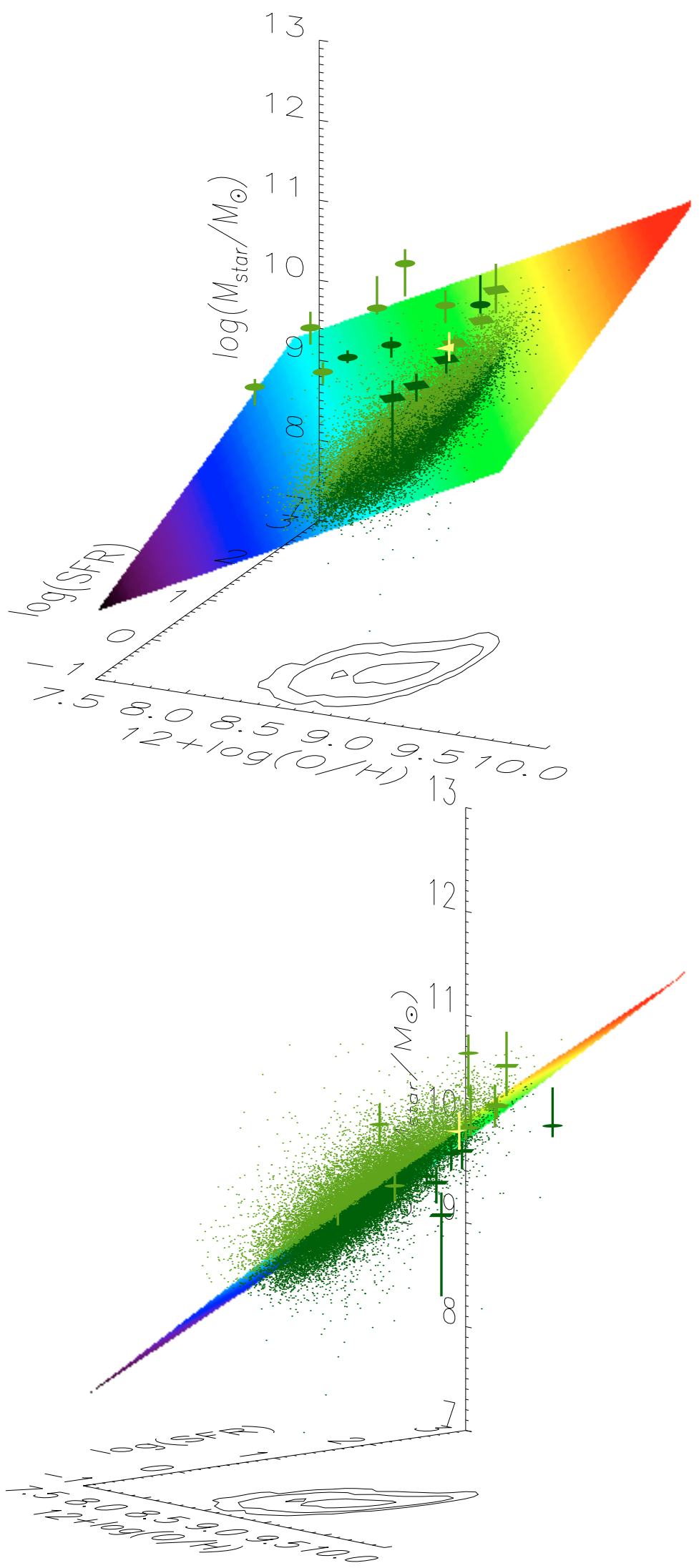

Fig. A.1. Tridimensional representation of the FP according to the fit given in Eq. (1), the color scale of the plane is related with the stellar mass. Light color symbols are located above the plane, while the dark ones are below it. Dots represent the SDSS local sample described in Sect. 2, diamonds correspond to the Erb et al. (2006) data at $z \sim 2.2$, and circles symbolise the data at $z \sim 3.5$ from Maiolino et al. (2008). The yellow triangle represents the data of Rodrigues et al. (2008) at $z \sim 0.85$. The iso-density contours are subject to a projection of the SDSS data cloud onto the metallicity-SFR plane, and they are given only as a visual aid. 